\title{
Clinical Characteristics of Patients with Mycoplasma pneumoniae-Related Acute Hepatitis
}

\author{
Su Rin Shin Sang Hoon Park Joo-Hee Kim Jun-Wook Ha Yu Jin Kim \\ Sung Won Jung Jin Bae Kim Myung Seok Lee Choong Kee Park \\ Department of Internal Medicine, Hallym University Medical Center, Seoul, Korea
}

\section{Key Words}

Mycoplasma pneumoniae $\cdot$ Hepatitis $\cdot \mathrm{C}$-reactive protein

\begin{abstract}
Background/Aims: Although the hepatobiliary manifestations of Mycoplasma pneumoniae infection have been described in several previous studies, controversies still remain. The aim of this study was to evaluate the clinical characteristics of patients with M. pneumoniae-related hepatitis and compare to those with M. pneumoniae infection but not hepatitis. Methods: We reviewed the medical chart of patients diagnosed with M. pneumoniae infection serologically. Results: Among the 117 patients with M. pneumoniae infection enrolled in the study, 25 showed acute hepatitis without any evidence of another cause. Patients with hepatitis presented with prodromal symptoms more frequently and also had a higher body temperature and C-reactive protein (CRP) levels than patients without hepatitis. Frequencies of respiratory tract involvement were not different between two groups. Clinical signs recovered within three weeks in both groups following the institution of antibiotic therapy. Multivariate analysis revealed that male sex, age $<40$ years, and high CRP levels were significantly linked to $M$. pneumoniae-related hepatitis. Conclusions: We found that acute hepatitis associ-
\end{abstract}

ated with M. pneumoniae occurred in about $21 \%$ of all $M$. pneumoniae infections, and gender, age, and CRP levels were factors related to the occurrence of $M$. pneumoniae-related hepatitis.

Copyright $\odot 2012$ S. Karger AG, Basel

\section{Introduction}

Although Mycoplasma pneumoniae infection usually affects the respiratory tract in older children and young adults, extrapulmonary involvement may also occur, including hematopoietic, cardiovascular, central nervous, musculoskeletal, cutaneous, gastrointestinal, hepatobiliary, genitourinary system, and occasionally multiorgan disease $[1,2]$. Among these, simultaneous elevations of liver enzymes associated with $M$. pneumoniae infection have been reported to occur in $50 \%$ of patients with $M$. pneumoniae-induced pneumonia [3]. However, most previous studies of $M$. pneumoniae-related hepatitis were based on a small case series and the range of incidences reported varies widely.

Therefore, in this study, we estimated the incidence of M. pneumoniae-related hepatitis and its clinical characteristics based on a large patient series. 


\section{Materials and Methods}

\section{Patients}

We reviewed the medical records of 163 patients who were diagnosed with $M$. pneumoniae infection at Hallym and Kangnam Sacred Heart Hospitals affiliated with Hallym University between February 2006 and May 2010.

Among patients who were diagnosed with serologically-confirmed $M$. pneumoniae infection, those who were older than 15 years of age and had serum alanine aminotransferase (ALT) or alkaline phosphatase (ALP) levels 2-fold greater than the upper normal limits were regarded as having hepatitis. Patients with various conditions that could cause liver function test (LFT) abnormalities were excluded; documented evidence of acute or chronic viral hepatitis, a habitual alcohol intake $>30 \mathrm{~g} / \mathrm{day}$, conditions highly suspicious of non-alcoholic fatty liver disease by abdominal imaging study or clinical factors including obesity (body mass index $>25$ ), diabetes, and dyslipidemia, history of drugs reported to be hepatotoxic, those who were taking complementary or alternative medicines within recent 6 months, infectious disease known for involving the liver, Wilson's disease, hemochromatosis, autoimmune hepatitis, or other infiltrative diseases.

We compared the clinical characteristics, laboratory data, and disease courses of $M$. pneumoniae-infected patients with and without hepatitis.

\section{Laboratory Evaluation}

To confirm M. pneumoniae infection, we used the Serodia Mayo II microparticle agglutination (MAG) assay (Fujirebio, Tokyo, Japan), which is a more advanced alternative to the complement fixation test for the detection of M. pneumoniae-specific antibody. Sensitivity and specificity of the MAG test for diagnosing M. pneumoniae infection has been reported as 10-65 and 80$88 \%[4,5]$. LFT including assessment of aminotransferase and ALP levels were performed using an automated chemistry analyzer (Hitachi $7600^{\circledR}$; Hitachi High-Technologies, Tokyo, Japan).

\section{Definition}

Because the enrolled patients were older than the prevalent population, $M$. pneumoniae infection was diagnosed based on a highly elevated $M$. pneumoniae titer $\geq 1: 320$ as measured by the MAG test on the basis of a single antibody determination. In cases with a lower level of titer, a second sample was examined after an interval of 2-3 weeks to confirm a 4 -fold titer increase [6].

The ratio of serum ALT (as a multiple of its upper normal limit) to serum ALP (as a multiple of its upper normal limit) has been designated as the $\mathrm{R}$ (for ratio) value. 'Hepatocellular' pattern was defined as $\mathrm{R} \geq 5$, 'cholestatic' as $\mathrm{R} \leq 2$, and 'mixed' as $\mathrm{R}>2$ to $\mathrm{R}<5$ [7].

Patients were categorized according to the time interval from the symptom onset to the peak LFT abnormalities. A time interval $<7$ days was categorized as early-onset hepatitis and an interval $\geq 7$ days as late-onset hepatitis [8].

\section{Medical Ethics and Statistics}

This study was performed according to the guidelines of the Declaration of Helsinki and the study protocol was approved by the institutional review board of Hallym and Kangnam Sacred Heart Hospitals. The significance of differences between groups was evaluated using the Mann-Whitney U test or Pearson's $\chi^{2}$ test.

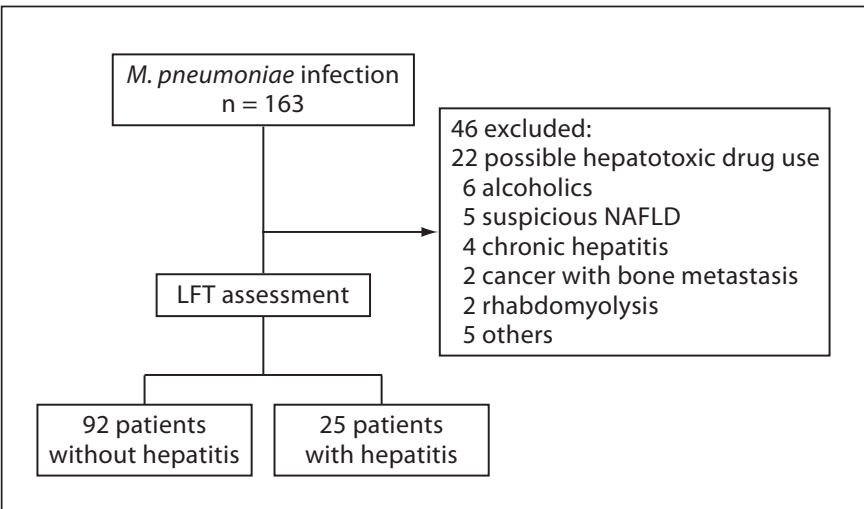

Fig. 1. Flow chart showing patient enrollment. NAFLD $=$ Nonalcoholic fatty liver disease.

All data are expressed as means \pm SDs with ranges for continuous variables and numbers with percentages for categorized variables unless indicated otherwise. Binary logistic regression analysis was used to determine factors significantly associated with $M$. pneumoniae-related hepatitis. Differences were considered significant when the $\mathrm{p}$ value was $<0.05$.

\section{Results}

\section{Demographic Characteristics of Patients with}

\section{M. pneumoniae Infection}

Figure 1 indicates the disposition of patients. The list of hepatotoxic drugs included antituberculous drugs in 10 patients, antibiotics in 5 ( 2 cephalosporins, 2 quinolones, and 1 amoxicillin), non-steroidal anti-inflammatory drug-containing common cold medicines in 2 , alternative medicines in 2, antiepileptic drug in 1 , and antifungal in 1, and allopurinol in 1 patient. Five cases designated 'others' had had end-stage renal disease, leukemia, a recent episode of seizure, cardiopulmonary resuscitation, and ischemic heart disease with cardiac enzyme elevation. After exclusion, 25 patients were diagnosed with M. pneumoniae-related hepatitis. Patients of all ages were included. We found that patients with hepatitis were younger than those without hepatitis but statistical significance was not observed. Male sex was significantly associated with the development of hepatitis and constitutional symptoms including fever and headache developed more frequently in patients with hepatitis than those without. Common presentations of $M$. pneumoniae infection were fever and cough, and the illness was often confined to the upper respiratory tract as a mild pharyn- 
Table 1. Demographic and clinical characteristics of the enrolled patients

\begin{tabular}{|c|c|c|c|c|}
\hline Variables & $\begin{array}{l}\text { All patients } \\
(\mathrm{n}=117)\end{array}$ & $\begin{array}{l}\text { Without hepatitis } \\
(\mathrm{n}=92)\end{array}$ & $\begin{array}{l}\text { With hepatitis } \\
(\mathrm{n}=25)\end{array}$ & $\mathrm{p}$ value \\
\hline Age, years & $45 \pm 38$ & $47 \pm 23$ & $38 \pm 19$ & 0.150 \\
\hline Male sex, $\mathrm{n}$ & $53(45)$ & $37(40)$ & $16(64)$ & 0.034 \\
\hline Body mass index & $24 \pm 19$ & $24 \pm 22$ & $23 \pm 3$ & 0.455 \\
\hline \multicolumn{5}{|l|}{ Underlying disease } \\
\hline Chronic lung disease & $23(20)$ & $21(23)$ & $2(8)$ & 0.154 \\
\hline Hypertension & $17(15)$ & $15(16)$ & $2(8)$ & 0.522 \\
\hline Diabetes & $6(5)$ & $4(4)$ & $2(8)$ & 0.607 \\
\hline Ischemic heart disease & $6(5)$ & $5(2)$ & $1(4)$ & 1.000 \\
\hline Malignancy & $5(4)$ & $5(5)$ & 0 & 0.583 \\
\hline Onset, days & $8.2(1-30)$ & $8.5(1-30)$ & $7.4(1-28)$ & 0.251 \\
\hline \multicolumn{5}{|l|}{ Symptoms } \\
\hline Fever & $93(80)$ & $69(75)$ & $24(96)$ & 0.021 \\
\hline Headache & $35(30)$ & $22(24)$ & $13(52)$ & 0.007 \\
\hline Myalgia & $34(29)$ & $21(23)$ & $13(52)$ & 0.005 \\
\hline Cough/sputum & $82(70)$ & $66(73)$ & $16(64)$ & 0.407 \\
\hline Chest discomfort & $19(16)$ & $16(18)$ & $3(12)$ & 0.761 \\
\hline Abdominal pain & $12(10)$ & $8(9)$ & $4(16)$ & 0.286 \\
\hline \multicolumn{5}{|l|}{ Radiologic findings } \\
\hline Pneumonic consolidation & $68(58)$ & $52(56)$ & $16(64)$ & 0.502 \\
\hline Pleural effusion & $22(19)$ & $14(15)$ & $8(32)$ & 0.081 \\
\hline Body temperature, ${ }^{\circ} \mathrm{C}$ & $37.9 \pm 1.1$ & $37.8 \pm 1.1$ & $38.6 \pm 1.0$ & 0.002 \\
\hline Season of admission & & & & 0.097 \\
\hline Spring & $36(31)$ & $28(30)$ & $8(32)$ & \\
\hline Summer & $36(31)$ & $28(30)$ & $8(32)$ & \\
\hline Autumn & $28(24)$ & $22(24)$ & $6(24)$ & \\
\hline Winter & $17(15)$ & $14(15)$ & $3(12)$ & \\
\hline \multicolumn{5}{|l|}{ Time interval to peak LFT } \\
\hline abnormality, days & & & $9.9 \pm 8.8$ & \\
\hline
\end{tabular}

Values in parentheses are percentages or ranges. LFT $=$ Liver function abnormality.

gitis or bronchitis. A coarse breathing sound was the most frequent clinical finding on physical examination, while skin rashes, cervical lymphadenopathy, or right upper quadrant tenderness were rarely observed. The patients with hepatitis had a significantly higher body temperature than those without. Although the trend that pleurisy was more common in the patients with hepatitis than those without was observed, statistical significance was not proven. A decreased number of patients were diagnosed with hepatitis in winter, but there were no significant seasonal variations in the incidence of diagnosis between patients with and without hepatitis (table 1).

The mean time from symptom onset to peak LFT abnormalities was 9.9 days. Twelve patients were classified as 'early-onset hepatitis' (median 4 days, range 1-6 days) and 13 were 'late-onset hepatitis' (median 13 days, range 7-38 days).

\section{Laboratory Findings}

On initial evaluation, complete blood counts were within normal ranges in both groups, but there was a shift to less mature cells in the hepatitis group. Although serum albumin levels were lower in patients with hepatitis, this difference was not clinically significant. Of 25 patients who presented with hepatitis, 12 had a hepatocellular pattern, 6 a cholestatic pattern with 1 hyperbilirubinemia, and 7 patients had a mixed pattern. Patients with hepatitis had a 4 -fold of mean increase in ALT levels (upper normal limit), and ALT levels were more than 5 -fold elevated in 10 of the hepatitis patients. C-reactive protein (CRP) levels were about 2 -fold higher in hepatitis patients than those infected with M. pneumoniae without hepatitis. One hundert and twelve patients were diagnosed by a single high titer of 1:320 or greater whereas 5 patients were diagnosed by 4 -fold or 
Table 2. Laboratory findings in patients with $M$. pneumoniae infection

\begin{tabular}{lcccc}
\hline Variables & $\begin{array}{c}\text { All patients } \\
(\mathrm{n}=117)\end{array}$ & $\begin{array}{l}\text { Without hepatitis } \\
(\mathrm{n}=92)\end{array}$ & $\begin{array}{l}\text { With hepatitis } \\
(\mathrm{n}=25)\end{array}$ & p value \\
\hline $\mathrm{WBC} / \mathrm{l}$ & $9,277 \pm 6,097$ & $9,522 \pm 6,518$ & $8,334 \pm 4,067$ & 0.551 \\
$\mathrm{PMN} / \mathrm{l}$ & $6,765 \pm 4,351$ & $6,949 \pm 4,440$ & $6,365 \pm 3,901$ & 0.544 \\
$\mathrm{Hb}, \mathrm{g} / \mathrm{dl}$ & $12.8 \pm 1.9$ & $12.8 \pm 1.9$ & $12.8 \pm 2.1$ & 0.919 \\
$\mathrm{PLT}, \times 10^{3} / \mu \mathrm{l}$ & $256 \pm 114$ & $266 \pm 118$ & $216 \pm 83$ & 0.156 \\
ALT, $\times$ UNL & $1.3 \pm 2.0$ & $0.5 \pm 0.3$ & $4.3 \pm 3.0$ & $<0.001$ \\
ALP, $\times$ UNL & $0.9 \pm 0.7$ & $0.5 \pm 0.3$ & $4.3 \pm 2.9$ & 0.008 \\
Total bilirubin, mg/dl & $0.7 \pm 0.5$ & $0.7 \pm 0.5$ & $0.8 \pm 0.7$ & 0.627 \\
Albumin, g/dl & $3.9 \pm 0.5$ & $4.0 \pm 0.5$ & $3.8 \pm 0.5$ & 0.026 \\
Creatinine, mg/dl & $1.0 \pm 0.8$ & $1.0 \pm 1.2$ & $0.8 \pm 0.2$ & 0.486 \\
CRP, mg/l & $84 \pm 82$ & $71 \pm 70$ & $140 \pm 103$ & 0.001 \\
Anti-Mycoplasma Ab & & & & 0.082 \\
$\quad 1: 160$ & $5(4)$ & $2(2)$ & $3(12)$ & $7(28)$ \\
$1: 320$ & $50(43)$ & $43(47)$ & $9(36)$ & \\
$1: 640$ & $37(32)$ & $28(30)$ & $6(24)$ & \\
$>$ or $1: 1,280$ & $25(21)$ & $19(20)$ & & \\
\hline
\end{tabular}

Values in parentheses indicate percentages.

$\mathrm{WBC}=$ White blood cells; PMN = polymorphonuclear cells; $\mathrm{Hb}=$ hemoglobin; PLT = platelets; ALT = alanine aminotransferase; $\mathrm{UNL}=$ upper normal limit; $\mathrm{ALP}=$ alkaline phosphatase; $\mathrm{CRP}=\mathrm{C}$-reactive protein.

greater rise of antibody titers after initial demonstration (table 2). The proportion of those who were diagnosed by serial increase was not significantly different between the two groups $(p=0.06)$.

There was no significant difference between late-onset hepatitis groups in all clinical and laboratory variables except CRP levels, which was significantly higher in the early-onset hepatitis group than in the late-onset hepatitis group (median 145 vs. $90 \mathrm{mg} / \mathrm{l}, \mathrm{p}=0.017$, data not shown).

\section{Clinical Course}

For treatment, macrolides were more frequently used to treat those with $M$. pneumoniae hepatitis than patients who did not have hepatitis ( 92 vs. $69 \%$, respectively). The mean duration of antibiotic treatment was not significantly different between the two groups (10.6 vs. 9.3 days, $\mathrm{p}=0.477)$. LFT results were normal in all patients within 3 weeks after antibiotic treatment with the exception of 2 cases who were lost to follow-up. Acute kidney injury occurred in 2 patients without hepatitis, and myocarditis in 1 patient with hepatitis (table 3).

\section{Uni- and Multivariate Analyses}

We undertook logistic regression analysis to determine which factors were associated with $M$. pneumoniae-
Table 3. Treatment and clinical course of patients with M. pneumoniae infection

\begin{tabular}{lcccc}
\hline & $\begin{array}{l}\text { All } \\
\text { patients } \\
(\mathrm{n}=117)\end{array}$ & $\begin{array}{l}\text { Without } \\
\text { hepatitis } \\
(\mathrm{n}=92)\end{array}$ & $\begin{array}{l}\text { With } \\
\text { hepatitis } \\
(\mathrm{n}=25)\end{array}$ & $\begin{array}{l}\mathrm{p} \\
\text { value }\end{array}$ \\
\hline Antibiotics & & & & 0.051 \\
$\quad$ Macrolides & $96(82)$ & $63(69)$ & $23(92)$ & \\
$\quad \begin{array}{l}\text { Quinolones } \\
\text { Others }\end{array}$ & $28(24)$ & $26(28)$ & $2(8)$ & \\
$\begin{array}{c}\text { Duration of treat- } \\
\text { ment, days }\end{array}$ & $3(3)$ & $3(3)$ & $0(0)$ & \\
$\begin{array}{c}\text { Complications } \\
\text { Com }\end{array}$ & $3(3)$ & $2(2)$ & $1(4)$ & 0.517 \\
\hline
\end{tabular}

Values in parentheses are percentages.

related hepatitis. On univariate analysis, male sex, a body temperature of $38^{\circ} \mathrm{C}$ or more, and high CRP levels were significantly associated with $M$. pneumoniae hepatitis. After multivariate logistic regression analysis, male sex, age $<40$ years, and high CRP levels were significantly related to hepatitis caused by M. pneumoniae. On multivariate analysis, body temperature did not show any relationship to the development of hepatitis in contrast to the univariate analysis results (table 4). 
Table 4. Uni- and multivariate analyses of factors related to M. pneumoniae-induced hepatitis

\begin{tabular}{|c|c|c|c|c|c|c|c|c|c|c|}
\hline \multirow[t]{2}{*}{ Variables } & \multicolumn{5}{|c|}{ Univariate analysis } & \multicolumn{5}{|c|}{ Multivariate analysis } \\
\hline & B & $\mathrm{SE}$ & $\mathrm{p}$ value & Ex (B) & $95 \% \mathrm{CI}$ & B & SE & $\mathrm{p}$ value & $\mathrm{Ex}(\mathrm{B})$ & $95 \% \mathrm{CI}$ \\
\hline Sex, male & 0.972 & 0.468 & 0.038 & 2.643 & $1.056-6.610$ & 1.336 & 0.606 & 0.027 & 3.805 & $1.161-12.476$ \\
\hline Age $<40$ years & 0.662 & 0.466 & 0.155 & 1.939 & $0.778-4.834$ & 1.748 & 0.761 & 0.022 & 5.741 & $1.292-25.506$ \\
\hline $\mathrm{BT} \geq 38^{\circ} \mathrm{C}$ & 1.421 & 0.532 & 0.008 & 4.141 & $1.458-11.756$ & 0.933 & 0.637 & 0.143 & 0.396 & $0.729-8.863$ \\
\hline CRP, mg/l & 0.009 & 0.003 & 0.002 & 1.009 & $1.003-1.015$ & 0.012 & 0.004 & 0.003 & 1.012 & $1.004-1.019$ \\
\hline
\end{tabular}

$\mathrm{BT}=$ Body temperature; CRP $=$ C-reactive protein.

\section{Discussion}

Since $M$. pneumoniae infection presenting as acute hepatitis was first described in 1975, several authors have investigated the clinical presentation of $M$. pneumoniaerelated hepatitis. According to those reports, M. pneumoniae-related hepatitis occurs in 2-5\% of all M. pneumoniae infection cases $[9,10]$ and $11-50 \%$ of M. pneumoniae pneumonia cases $[1,3,11-13]$. The wide range of incidences of $M$. pneumoniae-related hepatitis reported may be due to the retrospective nature of these previous studies and/or the diverse definitions of hepatitis used in these different studies. The most well-recognized manifestation of M. pneumoniae-related hepatitis is elevation of ALT levels, while a few case reports of cholestatic hepatitis have been also documented [14-16]. In the current study, we observed that M. pneumoniae infection affected the hepatobiliary system of $21 \%$ of patients with $M$. pneumoniae infection. Most patients demonstrated an abnormal hepatocellular pattern, and as many as $40 \%$ of patients showed a sharp increase in ALT levels greater than 5 -fold the upper normal limit. The lower incidence of hepatitis among $M$. pneumoniae-infected patients in our study compared to that reported in previous studies may be due to strict patient selection criteria. We enrolled patients having at least more than 2-fold elevation in ALT or ALP levels because LFT is easily affected by numerous factors.

We also evaluated the factors related to hepatitis by $M$. pneumoniae. We found that M. pneumoniae-related hepatitis occurred more frequently in males, in relatively young patients, and those with high CRP levels. These results are consistent with previous reports that reported a higher prevalence of $M$. pneumoniae infection in male patients and more severe illness in these patients [17-20]. The propensity for male patients to develop M. pneumoniae-related hepatitis may indicate that males are more vulnerable to $M$. pneumoniae infection than females. It is well known that the severity of $M$. pneumoniae disease is related to the degree to which the host immune system responds to the infection, particularly with respect to extrapulmonary involvement [8]. Suzuyama et al. [1] documented that for M. pneumoniae-related hepatitis, inflammatory signs such as a higher body temperature, greater number of leukocytes, and elevated CRP levels were more likely to appear in patients with LFT abnormalities than those without. However, these authors included patients with liver function abnormalities at 2 weeks after treatment in their study, and did not perform any statistical analyses. Daxboeck et al. [12] investigated the clinical characteristics of patients with M. pneumoniae pneumonia with LFT abnormalities; they reported that these patients had higher leukocyte levels and CRP levels than the patients without LFT abnormalities even though statistical significance could not be proven in CRP levels (11.5 vs. $5.9 \mathrm{mg} / \mathrm{dl}$ ). They also reported that age or gender distribution was not significantly different between patients with and without ALT elevation. In that study, however, only 12 patients had elevated ALT levels. Despite the shortcomings of the previous studies, the results of those studies and ours suggest that an underlying immune response is involved in the development of $M$. pneumoniae-related hepatitis.

The pathogenesis of hepatitis induced by M. pneumoniae remains largely unknown, but several mechanisms have been proposed. One of the most intriguing theories is that immune-mediated damage, including autoimmunity in liver tissue, occurs after M. pneumoniae infection. The presence of cold agglutinin is a well-known feature of $M$. pneumoniae infection regardless of the mechanism by which antibodies are formed. This mechanism is also known to be involved in late-onset hepatitis (7-10 days after onset of fever) [8,21, 22]. Roelcke et al. [23] demonstrated that elevation of ALT levels for long 
periods of time may be mediated by immunological mechanisms, such as cross-reactive antibodies induced by $M$. pneumoniae interacting with sialo-oligosaccharides on hepatic cells. Another theory is direct invasion of the liver by the pathogen. Although infection of liver or biliary cells by M. pneumoniae has never been demonstrated, it has been shown that M. pneumoniae uses surface-associated pyruvate dehydrogenase to bind to the extracellular matrix protein, fibronectin, which is highly expressed in the liver [23]. Because the time interval from onset of symptoms to peak LFT abnormality might be helpful in speculating about the possible pathophysiologic mechanisms leading to M. pneumoniae-related hepatitis, we reclassified patients into either early- or late-onset hepatitis based on 7 days between onset of symptoms and peak LFT abnormalities. However, almost equal numbers of patients were classified into either category and this finding along with too small numbers of patients with $M$. pneumoniae-related hepatitis has made it impossible to make any speculations about possible mechanisms.

The patients of this study were older than 15 years, whereas M. pneumoniae infection mostly affects schoolaged children. Considering that adult patients with unexplainable LFT abnormalities and fever or cough/sputum are referred to the hepatology department, the results of this study provide useful clinical information that should be known to hepatologists. In fact, 9 patients with $M$. pneumoniae infection presented acute hepatitis without respiratory symptoms. Therefore, clinicians need to be vigilant about the possibility $M$. pneumoniae hepatitis when unknown liver function abnormalities are observed, even in the absence of respiratory symptoms. We found that patients with M. pneumoniae hepatitis had an excellent prognosis; no chronic hepatitis, liver failure, or death occurred. Similar to other M. pneumoniae infections, early detection and appropriate therapy can avert M. pneumoniae-related hepatitis, which can occasionally result in massive hepatocellular destruction and inflammatory infiltration [22, 24]. Interestingly, macrolides were more frequently used in patients with M.pneumoniae hepatitis than those without. Macrolides are metabolized in the liver and excreted primarily in the bile in contrast to quinolones that are metabolized via renal excretion $[10,25]$. This difference in use appears to be due to a different in preference for these two types of drug between hepatologists and pulmonologists.

Our study had some limitations. First, data were collected retrospectively. Cases of $M$. pneumoniae-related hepatitis may have been missed if the primary clinicians did not diagnose or suspect $M$. pneumoniae infection and did not perform the serologic test, because enrollment of this study was based on the results of Mycoplasma antibody test positivity. This could occur particularly when a respiratory symptom is minimal or absent. Second, the diagnosis was based on a serologic test only. Because of the fastidious growth requirements of M. pneumoniae, there are variable modalities for diagnosing $M$. pneumoniae. Among them, the MAG test has relatively high specificity but low sensitivity [26]. This could result in underdiagnosis, and the actual incidence of $M$. pneumoniae-related hepatitis is still unclear. Although a molecular assay combined with a serologic test could reduce the false-negative rate during the acute phase of $M$. pneumoniae infection or in immune-compromised hosts, commercial kits are lacking $[6,27]$. Third, the definition of $M$. pneumoniae-related hepatitis used in our study, at least a 2 -fold elevation in ALT or ALP levels, could be arbitrary. However, despite the possibility of selection bias, we do not believe that transient and equivocal liver function abnormalities have much clinical implication. Finally, we did not perform a histological assessment. To elucidate $M$. pneumoniae-related hepatitis, further studies including attention to liver histology are needed, as well as a survey of the clinical characteristics.

In conclusion, we observed significant hepatic involvement in about $21 \%$ of patients with $M$. pneumoniae infection, and some of these patients showed marked elevation of ALT levels (more than 5-fold the upper normal limit). Furthermore, we found that male gender, a relatively young age, and signs of inflammation were associated with hepatitis, which suggests that $M$. pneumoniae infection activates the host immune system. Well-designed cohort studies as well as additional case-control studies are required to estimate the exact incidence of $M$. pneumoniae hepatitis and to clarify the underlying mechanisms.

\section{Disclosure Statement}

The authors have no conflicts of interest to disclose. 


\section{References}

-1 Suzuyama Y, Iwasaki H, Izumikawa K, Hara $\mathrm{K}$ : Clinical complications of Mycoplasma pneumoniae disease - other organs. Yale J Biol Med 1983;56:487-491.

$>2$ Murray HW, Masur H, Senterfit LB, Roberts RB: The protean manifestations of $\mathrm{Myco}$ plasma pneumoniae infection in adults. Am J Med 1975;58:229-242.

-3 Squadrini F, Lami G, Pellegrino F, Pinelli G, Bavieri M, Fontana A, Bisetti A: Acute hepatitis complicating Mycoplasma pneumoniae infection. J Infect 1988;16:201-202.

$>4$ Liu FC, Chen PY, Huang FL, Tsai CR, Lee CY, Lin CF: Do serological tests provide adequate rapid diagnosis of Mycoplasma pneumoniae infection. Jpn J Infect Dis 2008;61:397-399.

$\checkmark 5$ Beersma MFC, Dirven K, Van Dam AP, Templeton KE, Claas ECJ, Goossens H: Evaluation of 12 commercial tests and the complement fixation test for Mycoplasma pneumoniae-specific immunoglobulin G ( IgG) and IgM antibodies, with PCR used as the 'gold standard'. J Clin Microbiol 2005;43: 2277.

$\checkmark 6$ Daxboeck F, Krause R, Wenisch C: Laboratory diagnosis of Mycoplasma pneumoniae infection. Clin Microbiol Infect 2003;9:263273.

$>7$ Lee SH, Jeong SH, Lee D, Lee JH, Hwang SH, Ae Cho Y, Park YS, Hwang JH, Kim JW, Kim $\mathrm{N}$ : An epidemiologic study on the incidence and significance of the mutations in a Korean cohort with nonalcoholic fatty liver disease. J Clin Gastroenterol 2010;44:e154.

$>8$ Narita M: Pathogenesis of extrapulmonary manifestations of Mycoplasma pneumoniae infection with special reference to pneumo nia. J Infect Chemother 2010;16:162-169. $\checkmark 9$ Ali NJ, Sillis M, Andrews BE, Jenkins PF, Harrison BD: The clinical spectrum and diagnosis of Mycoplasma pneumoniae infection. Q J Med 1986;58:241-251.

10 Mansel J, Rosenow E III, Smith T, Martin J Jr: Mycoplasma pneumoniae pneumonia. CHEST 1989;95:639-646.

11 Romero-Gomez M, Otero MA, SanchezMunoz D, Ramirez-Arcos M, Larraona JL, Suarez Garcia E, Vargas-Romero J: Acute hepatitis due to Mycoplasma pneumoniae infection without lung involvement in adult patients. J Hepatol 2006;44:827-828.

12 Daxboeck F, Gattringer R, Mustafa S, Bauer C, Assadian O: Elevated serum alanine aminotransferase levels in patients with serologically verified Mycoplasma pneumoniae pneumonia. Clin Microbiol Infect 2005;11: 507-510.

13 Nagayama Y, Sakurai N, Yamamoto K: Clinical observations of children with pleuropneumonia due to Mycoplasma pneumoniae. Pediatr Pulmonol 1990;8:182-187.

14 Grullich C, Baumert TF, Blum HE: Acute Mycoplasma pneumoniae infection presenting as cholestatic hepatitis. J Clin Microbiol 2003;41:514-515.

15 Arav-Boger R, Assia A, Spirer Z, Bujanover Y, Reif S: Cholestatic hepatitis as a main manifestation of Mycoplasma pneumoniae infection. J Pediatr Gastroenterol Nutr 1995; 21:459-460.

16 Hiew TM, Tan AM, Ong EK, Ho L: Unusual manifestations of Mycoplasma pneumoniae infection in children. Singapore Med J 1995; 36:293-298.

17 Foy HM, Grayston JT, Kenny GE, Alexander ER, McMahan R: Epidemiology of Mycoplasma pneumoniae infection in families. JAMA 1966;197:859.
18 Noah ND: Mycoplasma pneumoniae infection in the United Kingdom - 1967-1973. Br Med J 1974;2:544-546.

19 Markham JG: Mycoplasma pneumoniae infection in 1978. NZ Med J 1979;90:473-474.

20 Waites KB, Talkington DF: Mycoplasma pneumoniae and its role as a human pathogen. Clin Microbiol Rev 2004;17:697.

21 Fernald GW: Immunologic mechanisms suggested in the association of $M$. pneumoniae infection and extrapulmonary disease: a review. Yale J Biol Med 1983;56:475-479.

22 Kountouras D, Deutsch M, Emmanuel T, Georgiadis G, Koskinas J: Fulminant $\mathrm{Myco}$ plasma pneumoniae infection with multi-organ involvement: a case report. Eur J Intern Med 2003;14:329-331.

23 Roelcke D, Kreft H, Northoff H, Gallasch E: Sia-b1 and I antigens recognized by $\mathrm{Myco}$ plasma pneumoniae-induced human cold agglutinins. Transfusion 1991;31:627-630.

24 John SD, Ramanathan J, Swischuk LE: Spectrum of clinical and radiographic findings in pediatric Mycoplasma pneumonia. Radiographics 2001;21:121-131.

25 Mandell GL, Bennett JE, Dolin R: Mandell, Douglas, and Bennett's Principles and Practice of Infectious Diseases, ed 7. Philadelphia, Churchill Livingstone, 2009, pp 14371445.

26 Nir-Paz R, Michael-Gayego A, Ron M, Block $\mathrm{C}$ : Evaluation of eight commercial tests for Mycoplasma pneumoniae antibodies in the absence of acute infection. Clin Microbiol Infect 2006; 12:685-688.

27 Waites KB, Balish MF, Atkinson TP: New insights into the pathogenesis and detection of Mycoplasma pneumoniae infections. Future Microbiol 2008;3:635-648. 